\title{
Evaluation of the Demographic and Laboratory Data of Patients Diagnosed with Crimean-Congo Hemorrhagic Fever in the Emergency Department and Their Relationship with Morbidity and Mortality
}

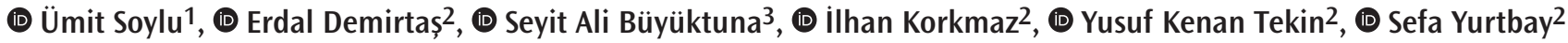 \\ ${ }^{1}$ Clinic of Emergency Medicine Service, Bozüyük State Hospital, Bilecik, Turkey \\ 2Department of Emergency Medicine, Sivas Cumhuriyet University Faculty of Medicine, Sivas Turkey \\ ${ }^{3}$ Department of Infectious Disease and Clinical Microbiology, Sivas Cumhuriyet University Faculty of Medicine, Sivas Turkey
}

\begin{abstract}
Aim: Crimean-Congo hemorrhagic fever (CCHF) is transmitted by infected ticks or through contaminated blood, tissue, and body fluids. Pathological laboratory results, such as thrombocytopenia, leukopenia, and anemia, along with biochemistry and coagulation parameters, can be used for its diagnosis and the determination of its prognosis.

Materials and Methods: Data of patients over 17 years of age diagnosed with CCHF between 2013 and 2018 were reviewed retrospectively. The complete blood count, liver-renal enzymes, electrolytes, prothrombin time, activated partial thromboplastin time (aPTT), D-dimer values, fibrinogen values, and international normalized ratio (INR) were recorded and analyzed at admission.

Results: Non-survivors had higher levels of alkaline phosphatase, alanine aminotransferase, amylase, aspartate aminotransferase (AST), blood urea nitrogen (BUN), direct bilirubin, gamma-glutamyl transferase, creatinine, potassium, total bilirubin and uric acid $(\mathrm{p}<0.05)$, whereas creatine kinase (CK), CK-MB, and calcium levels were lower $(p<0.05)$. Non-survivors had higher levels of basophil, mean corpuscular hemoglobin concentration, mean corpuscular volume, neutrophil, nucleated red blood cells, platelet distribution width, and white blood cells $(p<0.05)$.

Conclusion: Evaluation of routine blood parameters of CCHF patients in the emergency room is a useful tool to accelerate recovery in intensive care and prevent delay in patient treatment. Platelet, aPTT, INR, BUN, and AST values are predictors for mortality.

Keywords: Crimean-Congo hemorrhagic fever, white blood cell, thrombocyte, mortality
\end{abstract}

\section{Introduction}

Crimean-Congo hemorrhagic fever (CCHF) is one of the most common, fatal viral hemorrhagic fevers in the world. It is seen in many countries, especially in Asia, Africa, southeast Europe, and the Middle East (1). CCHF virus causes severe and fatal infection among vertebrae only in humans (2). The cause of CCHF is an RNA virus belonging to the Nairovirus genus from the Bunyaviridae family. The virus is transmitted to humans by infected ticks or by contact with contaminated blood, tissue, and body fluids. The risk of infection as a result of contact with infected blood is $8.7 \%$, especially during the follow-up of patients with bleeding. The risk rises to $33 \%$ in the case of needle stick injuries. The highest transmission is observed with a percutaneous injury. The fetal transmission has been reported to a small extent (3). The disease is seen in the spring, summer, and autumn seasons and peak in July (4). 
Thrombocytopenia is a severe laboratory finding in CCHF. The presence of severe thrombocytopenia in the early period can be considered as a clue that the disease may be fatal (5). The direct cytopathic effect of the virus is blamed for liver damage in the disease. Increased liver enzymes, edema in cells, and necrosis occur as a result of the CCHF virus infecting hepatocytes widely. As a result, bleeding occurs as a result of hemophagocytosis and liver dysfunction (6-9). Thrombocytopenia, leukopenia, and neutropenia occur in almost all cases. Severe patients may have anemia. Aspartate transferase (AST), alanine transferase (ALT), lactate dehydrogenase (LDH), and creatinine phosphokinase (CPK) levels are high. Total protein and albumin values may decrease, and prothrombin time (PT) and activated partial thromboplastin time (aPTT) among hemostasis tests are prolonged. Fibrinogen levels may decrease, and fibrin degradation products may increase. In severe patients, bilirubin, urea, and creatine values may increase $(10,11)$.

Using routine laboratory data in determining patients with fatal diseases such as CCHF will help the decision-making process of physicians. To our knowledge, there are studies on this topic in the literature, but there are limited studies with such a large number of cases. In this study, we aimed to investigate the laboratory results and demographic data of the patients who were diagnosed with CCHF in the last five years and to assess their roles in predicting mortality.

\section{Materials and Methods}

After the approval of the local ethics committee, the files of patients over the age of 17 who were diagnosed with CCHF between 2013 and 2018 were reviewed retrospectively. Ages, genders, and admission dates were recorded. The investigated admission laboratory parameters were as follows: fasting and postprandial blood glucose, alkaline phosphatase (ALP), ALT, amylase, AST, blood urea nitrogen (BUN), creatine kinase (CK), creatine kinase-MB (CK-MB), direct bilirubin, total bilirubin, gamma-glutamyltransferase (GGT), calcium $(\mathrm{Ca})$, chloride $(\mathrm{Cl})$, potassium $(\mathrm{K})$, sodium $(\mathrm{Na})$, creatinine, uric acid; basophil, eosinophil, hematocrit (HCT), hemoglobin (HGB), lymphocyte, mean corpuscular hemoglobin (MCH), mean corpuscular hemoglobin concentration (MCHC), mean corpuscular volume (MCV), monocyte, mean platelet volume (MPV), neutrophil, nucleated red blood cells (NRBC), plasma thromboplastin component (PTC), platelet distribution width (PDW), platelets (PLT), red blood cell (RBC), white blood cell (WBC); PT, aPTT, D-dimer, fibrinogen and international normalized ratio (INR). Patients with missing data were not included in the study.

\section{Statistical Analysis}

All statistical analyses were performed using IBM SPSS Statistics for Windows version 23.0 (IBM, Armonk, NY, USA), and continuous data were reported as mean \pm standard deviation or median, and categorical data were given as number and percentage. Kolmogorov-Smirnov test was used as a test for normality for continuous data, and parametric paired and unpaired t-tests were used. Receiver operating characteristic (ROC) analysis was used to determine the diagnostic efficiency of the laboratory parameters.

\section{Results}

Table 1 presents the gender distribution of patients with $\mathrm{CCHF}$ according to mortality. No statistically significant difference was observed between the two groups in terms of gender $(p>0.05)$. No statistically significant difference was observed in the withingroup evaluations. There was no effect of gender on mortality in CCHF patients. When the age was compared in terms of mortality, no statistically significant difference was found between the groups.

The distribution of patients according to the months of admission is given in Figure 1. The highest admission total was recorded as 134 patients in July. Again, the highest mortality was observed in July. No statistically significant difference was found in comparing the mortality of the disease by months $(p>0.05)$.

Table 2 shows the comparison of the laboratory data of the survivors and non-survivors. Regarding biochemistry parameters, non-survivors had higher levels of ALP, ALT, Amylase, AST, BUN, direct bilirubin, GGT, creatinine, $\mathrm{K}$, total bilirubin and uric acid levels and this was statistically significant $(p<0.05)$. In the non-

Table 1. Comparison of survivors and non-survivors in terms of gender

\begin{tabular}{|l|l|l|l|}
\hline Gender & Non-survivors (\%) & Survivors (\%) & p \\
\hline Female & $11(36.7 \%)$ & $191(41.3 \%)$ & $>0.05$ \\
\hline Male & $19(63.3 \%)$ & $271(58.7 \%)$ & \\
\hline Age & $\mathbf{p}>\mathbf{0 . 0 5}$ & $\mathbf{p}>\mathbf{0 . 0 5}$ & $>0.05$ \\
\hline
\end{tabular}




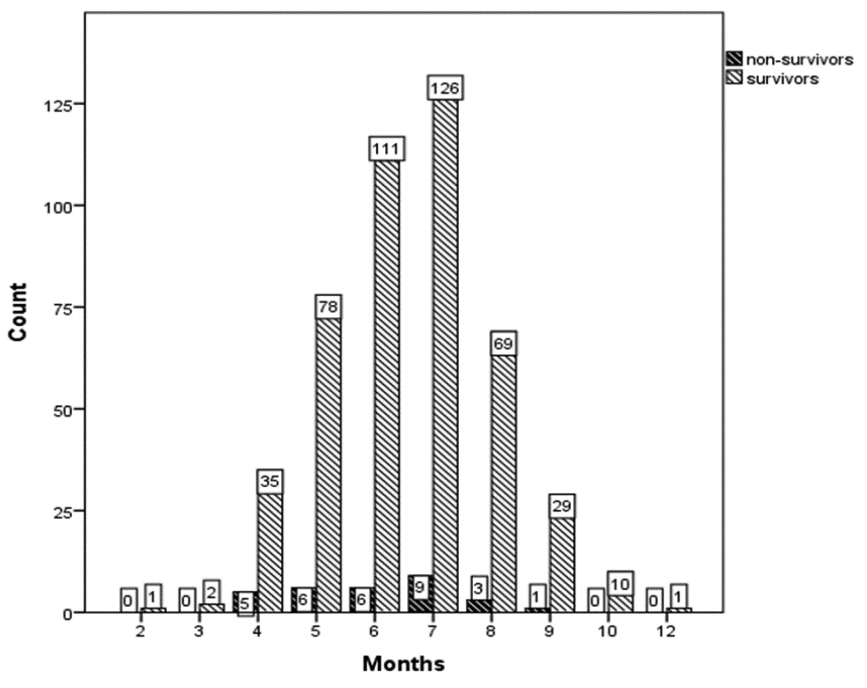

Figure 1. The distribution of patients according to the months of admission

survivor group, CK, CK-MB, and Ca levels were lower, and this was statistically significant $(p<0.05)$. No statistically significant difference was found in fasting and postprandial glucose levels, Na and $\mathrm{Cl}$ levels between two groups ( $\mathrm{p}>0.05)$. Regarding complete blood count (CBC) parameters, non-survivors had higher levels of basophil, MCHC, MCV, neutrophil, NRBC, PDW, and WBC, and this was statistically significant $(p<0.05)$. There was no statistically significant difference in terms of HCT, HGB, MCH, MPV, PDW, RBC, eosinophil, and lymphocyte count between two groups ( $p>0.05)$. In the non-survivor group, monocyte, PTC, and PLT levels were lower, and this was statistically significant $(p<0.05)$. Regarding coagulation parameters, non-survivors had higher levels of aPTT, D-dimer, and INR, and this was statistically significant $(p<0.05)$. In the non-survivor group, fibrinogen and PT levels were lower, and this was statistically significant $(p<0.05)$.

Table 3 shows the sensitivity, specificity, positive predictive values, negative predictive values, and area under curve values of the laboratory values of the patients according to the ROC analysis. AST and BUN from biochemical parameters, PLT from CBC parameters, and APTT and INR from coagulation parameters had the highest AUC parameters. Figure 2 shows the ROC analysis charts.

\section{Discussion}

Our study, in which we examined a large number of patient populations, revealed that ALT, AST, BUN, creatinine, CK-MB, aPTT, INR, PT, fibrinogen, and PLT could be functional determinants in demonstrating CCHF mortality.
In the studies conducted, no statistically significant difference was found between the survivors and non-survivors in terms of age and gender (12-14). In our study, in accordance with the literature, both groups show similar characteristics in terms of age and gender. Again, in our study, it was observed that the mortality was high in June and July when the disease was prevalent.

When the literature is reviewed, CBC parameters were evaluated in many studies. In a study of 152 patients with CCHF evaluated by Yilmaz et al. (15), admission HGB and PLT values were lower in the severe patient group than in the non-severe group, and this difference was significant (HGB cut-off: 13.5, AUC; 0.61, PLT cut-off: 90,000, AUC: 0.70). They stated that WBC was similar in both groups, and there was no statistical difference. In a study by Hatipoglu et al. (14), they examined the laboratory data of 152 CCHF patients as predictors of mortality. They found that HGB and WBC values were high in patients with mortality, and PLT values were low. In a study conducted by Arslan et al. (16), PLT and HGB values were found to be significantly lower in patients with bleeding compared to the group with no bleeding, and they did not find a statistically significant difference between WBC and CRP values. In the study by Öngörü et al. (12) in which they examined the relationship between coagulation parameters and mortality in CCHF patients, they found that PLT rates were significantly lower in the non-survivors. In the study of evaluating the laboratory and clinical features of CCHF patients as predictors of mortality, Kazancioğlu et al. (17) evaluated a total of 92 patients (77 survivors and 15 non-survivors). They found that the admission levels of HB and WBC did not differ significantly between the non-survivors and survivors. The admission PLT levels were found to be statistically significantly lower in the nonsurvivors. They found a cut-off value of $<30,000$ for admission PLT with $85.7 \%$ sensitivity and $81.8 \%$ specificity (AUC: 0.840 ). In the severity scoring system of CCHF developed by Bakır et al. (18), PLT decrease and WBC increase are included in the scoring system. In our study, we found many significant differences in CBC parameters, but according to ROC analysis, the PLT cut-off value of $<119,500$ was the most successful parameter with $79 \%$ sensitivity and $83 \%$ specificity. These values were also compatible with the literature. Studies reported different opinions with WBC increase. In our study, WBC was significantly higher in the nonsurvivor group. Again, there are conflicting opinions about HB in the literature. In our study, we found that admission HGB level was not significantly different between the two groups. We think that additional attention should be paid to the lower HGB levels in patients presenting with bleeding. 


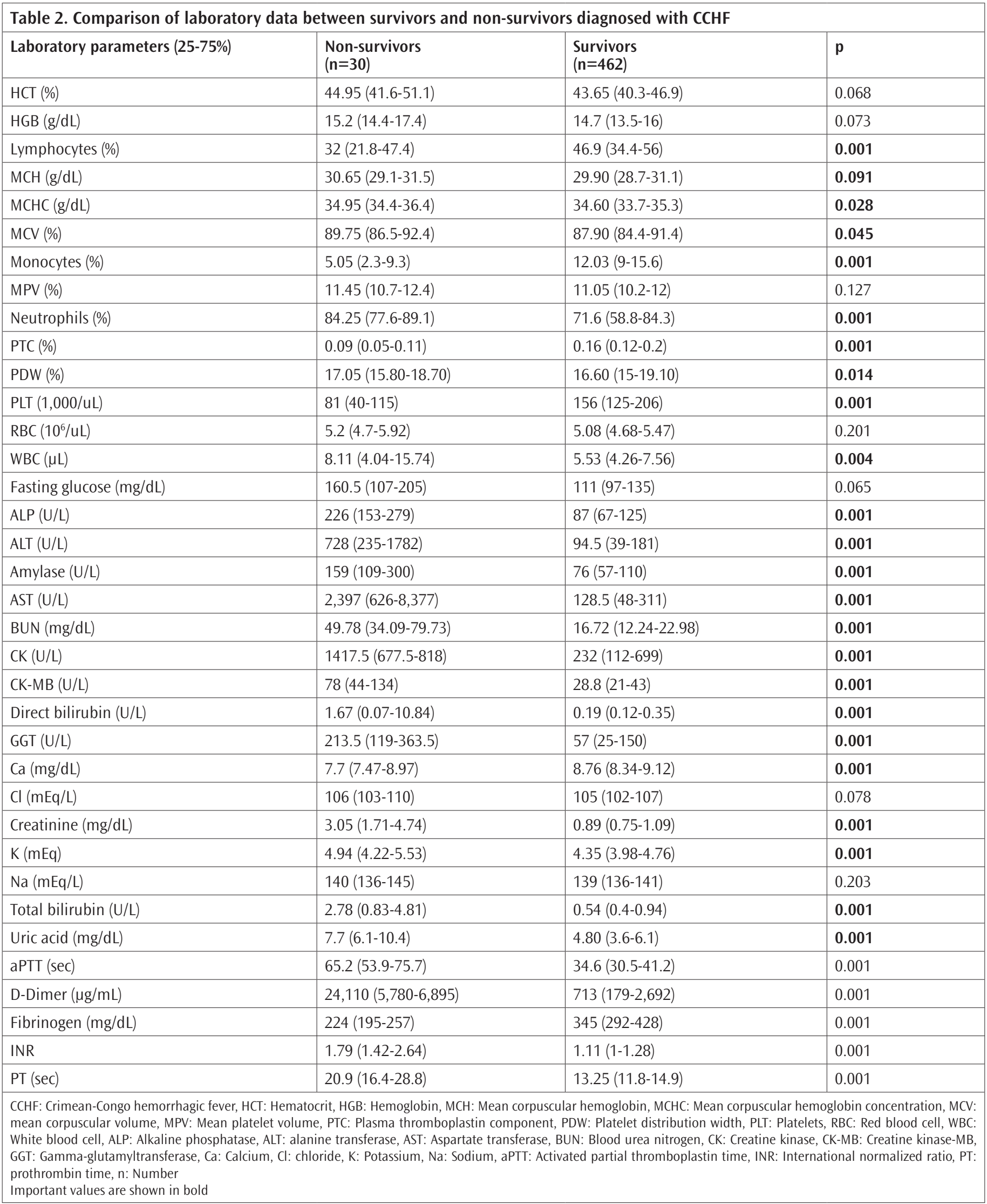




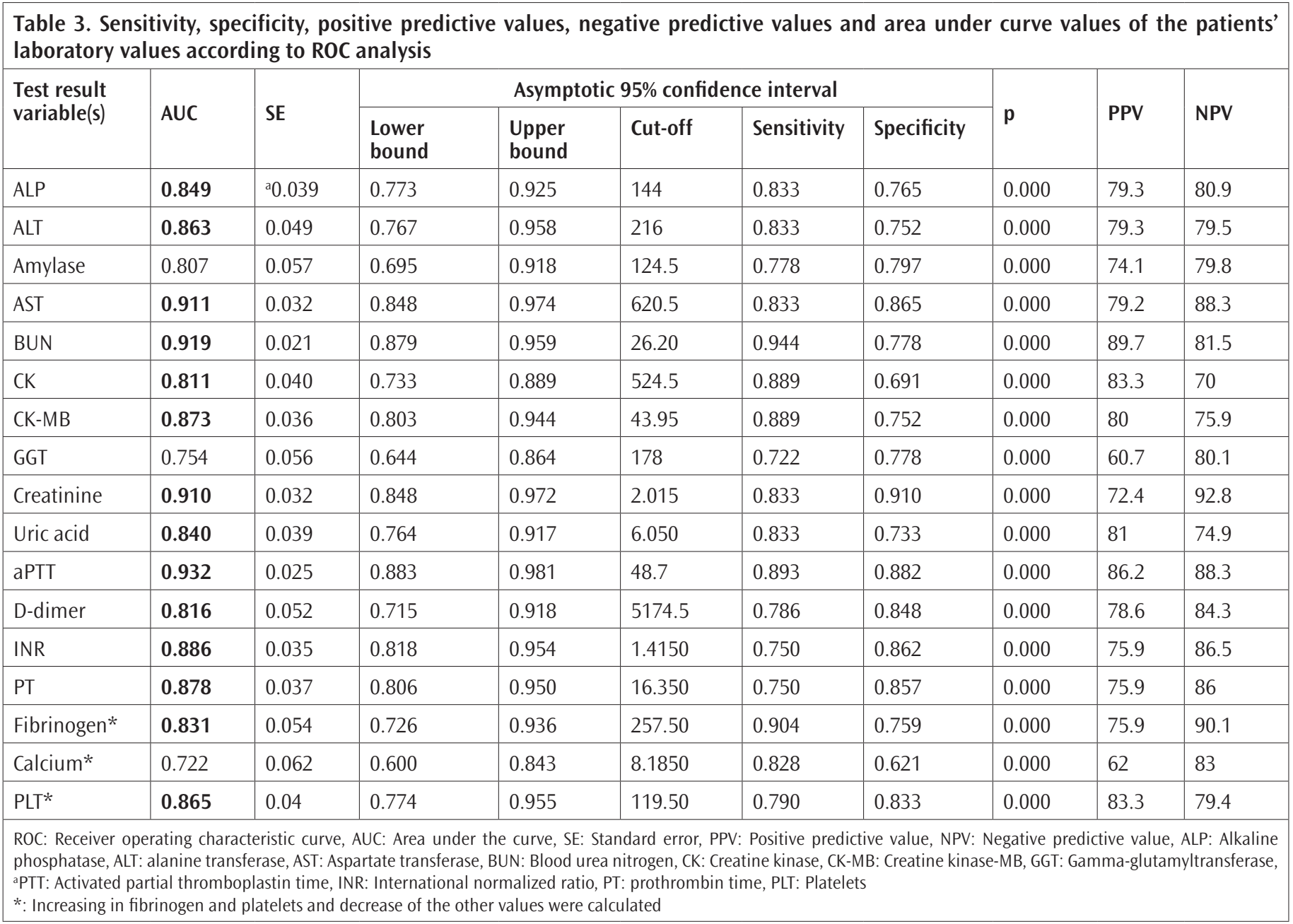

There are many studies examining biochemical parameters in CCHF. In these studies, especially AST, ALT, ALP, LDH values were found to be higher in severe patients $(12-14,16)$. In the study conducted by Yilmaz et al. (15), no statistically significant difference was found between BUN and creatinine levels between clinically severe and mild groups. In the study of Kazancioğlu et al. (17), admission urea and creatinine levels were found to be significantly higher in the non-survivor group. The sensitivity and specificity of urea for a cut-off value of 27.5 were $92.9 \%$ and $58.4 \%$, respectively ( $A \cup C=0.760$ ). Similar to these studies, in our study, AST, ALT, ALP, LDH, GGT, CK, CK-MB, BUN, creatinine, and uric acid were higher in the non-survivor group, and these were statistically different parameters. Especially BUN and AST were the biochemical parameters to be considered in the nonsurvivor group. BUN cut-off value of 26.2 had $94.4 \%$ sensitivity and $77.8 \%$ specificity. AST cut-off value of 620 had $83 \%$ sensitivity and $86 \%$ specificity. In another study, Ca levels in children with CCHF were found to be low (18). We also found that Ca levels were significantly lower in the non-survivor group.
Abnormal bleeding time and coagulation parameters can be counted as the most common laboratory findings in patients with CCHF. When we look at the studies in the literature, an increase in aPTT, PT, PTT, and INR, and a decrease in fibrinogen are observed (19-21). In our study, we also found PT, aPTT, INR, and D-dimer increase and fibrinogen decrease in accordance with the literature. Especially aPTT and INR elevation had the highest sensitivity and specificity among these parameters. The aPTT cut-off value of 48.7 had $89.3 \%$ sensitivity and $88.2 \%$ specificity. INR cut-off value of 1.41 had $75 \%$ sensitivity and $86 \%$ specificity.

\section{Conclusion}

Evaluation of routine blood parameters of CCHF patients in the emergency room is a useful tool to accelerate hospitalization in intensive care and to prevent delay in the treatment of the patient. It should be kept in mind that PLT, aPTT, INR, BUN, and AST values, especially in patients with suspected CCHF, are predictors of mortality. 

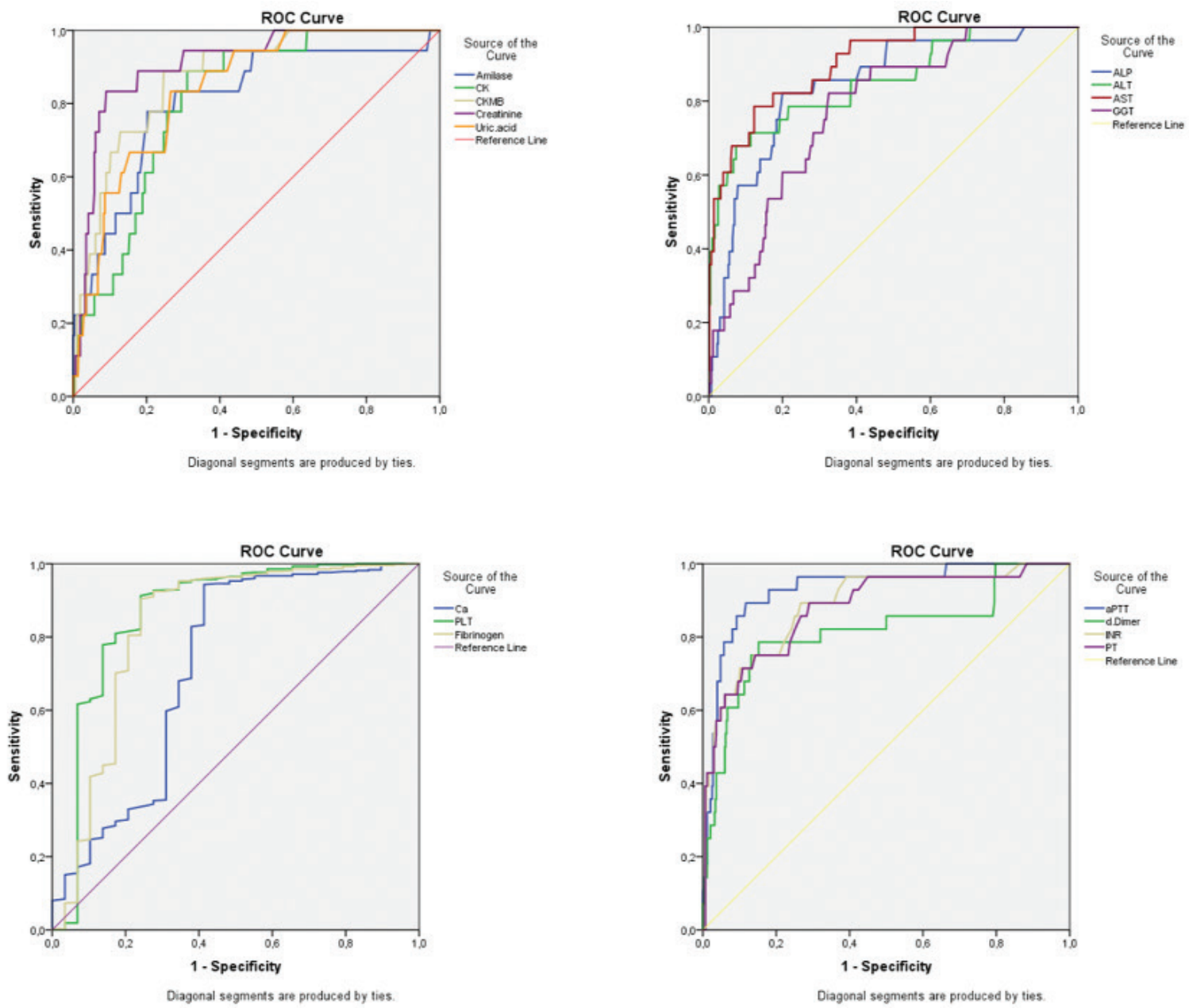

Figure 2. Shows the ROC analysis charts

ROC: Receiver operating characteristic curve, CK: Creatine kinase, CK-MB: Creatine kinase-MB, ALP: Alkaline phosphatase, ALT: Alanine transferase, AST: Aspartate transferase, aPTT: Activated partial thromboplastin time, INR: International normalized ratio, PT: Prothrombin time, PLT: Platelets

\section{Ethics}

Ethics Committee Approval: This retrospective study was approved by the institutional human ethics committee [Sivas Cumhuriyet University Ethics Committee (2018-11/06)].

Informed Consent: Retrospective study.

Peer-review: Externally peer-reviewed.

\section{Authorship Contributions}

Surgical and Medical Practices: Ü.S., E.D., S.A.B., Y.K.T., Concept: E.D., Y.K.T., Design: E.D., S.A.B., Y.K.T., Data Collection or Processing: I.K., Y.K.T., S.Y., Analysis or Interpretation: E.D., I..K., S.Y., Literature Search: E.D., S.A.B., S.Y., Writing: E.D., I..K.

Conflict of Interest: No conflict of interest was declared by the authors.
Financial Disclosure: The authors declared that this study received no financial support.

\section{References}

1. Ergönül Ö. Kırım Kongo kanamalı ateși. ANKEM Derg. 2009;23:234-40.

2. Bakır M. Kırım Kongo hemorajik ateși. ANKEM Derg. 2004;18:90-3.

3. Ergonul 0. Crimean-Congo haemorrhagic fever. Lancet Infect Dis. 2006;6:20314.

4. Bodur H. Kırım-Kongo kanamalı ateși ve DAS yönetimi. 5. Ulusal Sterilizasyon Dezenfeksiyon Kongresi; 2007 Nisan 4-8; Kundu, Antalya, Türkiye; 2007.p.509-20

5. Swanepoel R, Gill DE, Shepherd AJ, Leman PA, Mynhardt JH, Harvey S. The clinical pathology of Crimean-Congo hemorrhagic fever. Rev Infect Dis. 1989;11:794-800

6. Liu, Dongyou, editor. Manual of security sensitive microbes and toxins. CRC Press. 2014.p.37-52. 
7. Akıncı E, Bodur H, Leblebicioglu H. Pathogenesis of Crimean-Congo hemorrhagic Fever: Vector Borne Zoonotic Dis. 2013;13:429-37.

8. Bray M. Comparative pathogenesis of Crimean Congo hemorrhagic fever and Ebola hemorrhagic fever. In: Ergonul O, Whitehouse CA. Dordrecht, NL, editors. Crimean Congo Hemorrhagic Fever: A Global Perspective: Springer. 2007.p.221-31.

9. Ergonul O, Tuncbilek S, Baykam N, Celikbas A, Dokuzoguz B. Evaluation of serum levels of interleukin (IL)-6, IL-10, and tumor necrosis factor-alpha in patients with Crimean-Congo hemorrhagic fever. J Infect Dis. 2006;193:9414.

10. Özkurt Z. Kırım Kongo Kanamalı Ateși. Yoğun Bakım Derg. 2007;7:85-90.

11. Eren SH, Korkmaz I, Aktas C, Ay D. Diagnostic criteria in Crimean Congo haemorrhagic fever disease and cost analysis. Afr J Microbiol Res. 2010;4:6469.

12. Öngörü P, Dagdas S, Bodur H, Yilmaz M, Akinci E, Eren S, et al. Coagulopathy parameters in patients with Crimean-Congo Hemorrhagic fever and its relation with mortality. J Clin Lab Anal. 2010;24:163-6.

13. Karakecili F, Cikman A, Aydın M, Binay UD, Kesik OA, Özçiçek F. Evaluation of epidemiological, clinical, and laboratory characteristics and mortality rate of patients with Crimean-Congo hemorrhagic fever in the northeast region of Turkey J Vector Borne Dis. 2018;55:215-21.

14. Hatipoglu CA, Bulut C, Yetkin MA, Ertem GT, Erdinc FS, Kilic EK, et al. Evaluation of clinical and laboratory predictors of fatality in patients with Crimean-Congo haemorrhagic fever in a tertiary care hospital in Turkey. Scand J Infect Dis. 2010;42:516-21.
15. Yilmaz G, Koksal I, Topbas M, Yilmaz H, Aksoy F. The effectiveness of routine laboratory findings in determining disease severityin patients with Crimean-Congo hemorrhagic fever: Severity prediction criteria J Clin Virol. 2010;47:361-5.

16. Arslan M, Yilmaz G, Mentese A, Yilmaz H, Karahan SC, Koksal I. Importance of endothelial dysfunction biomarkers in patients with Crimean-Congo hemorrhagic fever. J Med Virol. 2017;89:2084-91.

17. Kazancioğlu S, Akinci E, Baştuğ A, Kayaaslan B, But A, Aslaner H, et al. Does the course of laboratory parameters help us to predict the outcome of CCHF. Turk J Med Sci. 2016;46:328-34.

18. Bakır M, Gözel MG, Köksal I, Așık Z, Günal Ö, Yılmaz H, et al. Validation of a severity grading score (SGS) system for predicting the course of disease and mortality in patients with Crimean-Congo hemorrhagic fever (CCHF) Eur J Clin Microbiol Infect Dis. 2015;34:325-30.

19. Uysal EB, Tuzcu N, Sancakdar E, Guven AS, Akkar I, Deveci K, et al. Vitamin D status in children with Crimean-Congo hemorrhagic fever. J Vector Borne Dis. 2015;52:36-9.

20. Whitehouse CA. Risk Groups and Control Measures for Crimean Congo Hemorrhagic Fever. Ergonul O, Whitehouse CA, editors. Crimean Congo Hemorrhagic Fever: A Global Perspective. Dordrecht (NL): Springer. 2007.p.273-80.

21. Ergönül Ö. Crimean-Congo hemorrhagic fever virus: newoutbreaks, newdiscoveries. Curr Opin Virol. 2012;2:215-20. 